\title{
As funções da leitura para graduandos e pós-graduandos
}

\author{
Gracieli Grizzi Lopes de Mendonça ${ }^{1}$ \\ S.E.C. - Pb - Bolsista CAPES
Maria Helena Mourão Alves Oliveira PUC-Campinas

\author{
Marcelo de Almeida Buriti \\ E.T.F.Pb. - Bolsista CNPq
}

\author{
Marisa Bueno Mendes Gargantini \\ PUC-Campinas
}

\begin{abstract}
O trabalho visa conhecer as funções da leitura identificadas pelos estudantes de graduação e pósgraduação, comparando-os com referência ao uso destas. São sujeitos da pesquisa 25 estudantes (11 alunos da pós-graduação e 14 da graduação). Utiliza como instrumento a escala das funções da leitura. Os resultados mostram que os estudantes utilizam-se de todas as funções, com uso predominante das funções Aprendizagem, Utilidade e Lazer. Aparece com bom índice de identificação Estímulo e Alvos Sociais Definidos. Fica evidente não haver diferença significante entre os dois grupos, o que permite questionar o desempenho da universidade na formação de sujeitos leitores. Palavras-chave: Funções da Leitura, estudantes de graduação, estudantes de pós-graduação.
\end{abstract}

\begin{abstract}
The functions of reading for undergraduate and graduate students.

The present work aims to study the functions of reading identified by undergraduate and graduate students by comparing such students according to the use they make of such functions. The sample consisted of 25 students ( 11 graduates and 14 undergraduates). The instrument used for this research was the functions of reading scale. The results of this study demonstrate that the students use all functions, being predominant the use of learning, utility, and leisure functions. Stimulus and definite social aims were significantly identified. There is no evidence of significant difference between the two groups. Such fact allows us to question the university's performance in producing readers.
\end{abstract}

Key-words: Reading Functions, Undergraduate Students, Graduate Students.

\section{Introdução}

A sociedade atual é marcada pelo rápido crescimento o qual desafia a capacidade humana a encontrar soluções para adequação entre os indices de crescimento e de desenvolvimento. Neste contexto, as organizações humanas tornam-se pontos de referência na geração das mudanças e na criação de processos adaptativos a estas, visando a própria sobrevivência.

1. Os autores agradecem a orientação da $\operatorname{Prof}^{\mathrm{a}} \mathrm{Dr}^{\mathrm{a}}$ Geraldina Porto Witter.

Endereço para correspondência: Rua Nazaré Paulista, 865, CEP 13094-610, Campinas, SP.
Das organizações, algumas foram criadas para desenvolver, induzir e favorecer transformações (Granja,1995). Dentre estas, destaca-se a Universidade que deve se voltar para uma instrução que oriente o aluno a considerar a interdependência dos fatos, formando assim sujeitos abertos para o conhecimento novo por meio da transação sociocultural. Este processo requer do sujeito uma consciência crítica e reflexiva capaz de orientá-lo entre o "conhecer" a realidade e o "saber responder" questões sobre esta realidade. A marca divisória entre estas duas posições se estabelece pelo construir um conhecimento, o qual tem carac- 
terísticas sociais e históricas que requerem, para sua propagação e apreensão, o uso da escrita e da leitura.

A informatização da vida social tem gerado a necessidade de efetivar mudanças nos processos de aprendizagem ao mesmo tempo que tem proposto questões sobre a estruturação do sistema de ensino. Um exemplo desta necessidade é descrito por Kulik e Kulik (apud Wepner, 1990) no estudo de meta-análise sobre a efetividade do uso do computador no desempenho de leitura. Os autores identificaram que a maioria dos estudos focalizados evidenciava um crescimento no nível do desempenho de leitura e uma diminuição no tempo instrucional requerido para a aprendizagem dessa habilidade. Assinalaram, também, que o uso do computador em sala de aula reforça as atividades didáticas que podem ser completadas rápida e facilmente, o que irá permitir aos estudantes mais tempo para a prática da leitura em suas diferentes formas. Porém, a efetividade deste trabalho exige alguns pré-requisitos relacionados à própria montagem do laboratório de informática e ao planejamento para a incorporação deste equipamento nos cursos das universidades. Isto implicará a preparação de instrutores habilitados não só no trabalho com "softwares", mas também com as estratégias e os procedimentos que propiciam a aprendizagem. Também os estudantes devem estar familiarizados com os mecanismos de "softwares" a fim de que hábitos produtivos de trabalho sejam empregados tão rapidamente quanto possível.

Contudo, tal mudança não é simplesmente tecnológica, pois requer também uma revisão no modo de organização e nas relações interpessoais. Com isso, pretende-se dizer que essas inovações tendem a valorizar de forma mais acentuada as habilidades mentais, cognitivas e comportamentais (Fidalgo,1994).
A leitura tem sido considerada como um processo interativo entre o leitor/texto/autor. "É a decodificação de símbolos gráficos e a transformação dos mesmos em unidades significativas (representativas) através do conhecimento e domínio da relação dos critérios de coesão e coerência textual " (Carnio,1995, p.9).

Ao ler um texto, o leitor tem um papel ativo na reconstrução do conteúdo do mesmo, apoiando-se em sua experiência prévia, em seus conhecimentos teóricos e, também, tomando por base os propósitos do autor e os elementos lingüísticos que possibilitam a expressão das idéias pretendidas.

Os extraordinários progressos tecnológicos, em matéria de comunicação, reforçam que a habilidade de ler continua a ser um componente fundamental para todo e qualquer tipo de aprendizagem. Assim, a leitura torna-se um comportamento imprescindivel na formação e no desenvolvimento do homem moderno. O domínio funcional da língua é desenvolvido por meio de processos de interação social e lingüística e não apenas através da exposição à linguagem, o que sugere uma transação social e cultural (Rosemblat, 1985 apud Dolly, 1990). É por meio desta relação que se desenvolvem os diferentes significados dos objetos, estados e processos que ocorrem no meio. Estabelece-se uma parceria entre autor-texto-leitor, embora muitos leitores não estejam conscientes disto (Goodman, 1983 apud Dolly, 1990). Não consideram a possibilidade de questionar ou responder ao texto escrito ou ao escritor talvez porque, como diz este autor, não vejam a leitura e a escrita com função genuína de comunicação.

Na relação pessoal-social, o hábito de leitura forma pessoas participantes e responsáveis pelo desenvolvimento sociocultural. Nesta posição, o sujeito leitor atualiza alguns motivos psicológicos entre os quais auto-respeito, autorealização, segurança e sucesso, necessidades 
que o direcionam para uma leitura específica e que, ao atender e satisfazer o motivo desencadeador, transformam-se em nova energia motivacional (Oliveira,1993). Este nível de leitor pressupõe uma relação "transacional" com o meio, uma capacidade de ajustar suas estratégias de leitura ao tipo de texto e informação veiculada. Esta capacidade garante um nível de compreensão que estará diretamente relacionado à possibilidade de reconhecer os vocábulos, estabelecer inferências e manter o constante relacionamento com a marca (gráfica e de estrutura) do texto (Oakhill e Gargham, 1988).

Tal processo transacional, ao mesmo tempo que fornece ao leitor base para compreensão e uso das funções da leitura, recebe dele novos dados referentes a seus motivos e necessidades, o que favorecerá a discussão e a formulação de outras funções.

Cabe, pois, a cada ser humano encontrar na leitura um motivo, uma ajuda para o seu próprio desenvolvimento. Este é o comportamento esperado tanto do corpo docente como discente de uma Universidade (Santos, 1990).

Além disso, como assinala Witter (1989), a leitura constitui-se um meio de acesso ao saber humano produzido em cada área do conhecimento, o que garantirá a recuperação da informação e a necessária atualização para a prática profissional eficiente. Isto significa que cada leitor deve reconhecer e identificar seus interesses e motivos para a leitura, usando-a para a efetivação das funções a ela relacionadas.

Gray (1984) listou alguns destes motivos que refletem uma preocupação com manter-se informado a fim de propiciar a definição de motivos profissionais precisos. Com isto, o leitor garantirá o seu sucesso pessoal, desenvolverá o próprio conhecimento e experimentará situações de prazer.

As diferenças culturais, conforme relatado por Fillmore (1981 apud Field, 1990), de- finem diferenças cognitivas que se refletirão nos processos interativos com a leitura e que atuarão, também, sobre os objetivos e as funções da leitura.

Atribui-se à leitura algumas funções que se relacionam à realização pessoal, incluindo-se os aspectos estéticos, afetivos e criativos. Além dessa dimensão pessoal, as funções têm relação com os aspectos sociais que orientam e reforçam todo o comportamento humano no atendimento de suas necessidades, perspectivas e realizações.

Greaney (1990), com base em de um estudo transcultural, listou dez funções da leitura: Aprendizagem, Lazer, Estímulo, Utilidade, Alvos Sociais Definidos, Moralidade, Flexibilidade, Auto-Respeito, Fuga, Preencher o Tempo, que variam conforme a região em que o leitor vive.

Em pesquisa de Oliveira (1993), voltada para a identificação das funções priorizadas por estudantes universitários, fica evidente o predomínio das funções "aprendizagem" e "utilidade". Embora possam estar de acordo com o momento de vida dos estudantes, a pouca atenção dada à leitura com função de formação da cidadania evidencia uma formação inadequadamente dirigida, comprometendo a diversidade necessária a uma formação integral. Isto reflete um leitor dependente, tendo em vista atender a uma necessidade específica.

Confirmando esta situação, a função "flexibilidade", que reflete a habilidade de leitura de diferentes tipos de texto, apresenta-se com baixo nível de utilização. Tal falta de flexibilidade compromete a leitura independente e a própria leitura atendendo à função "lazer", que parece, para o estudante, ter uma conotação negativa (Oliveira,1993).

A análise da distribuição das funções em nível de utilização demonstrou que os sujeitos pesquisados por Oliveira (1993, p.71) 
“incluem-se no nível médio de utilização (das funções) caracterizando um escore inferior ao esperado em universitários".

Os resultados obtidos por Lopes e col. (1992) confirmam dados anteriores, considerando-se que os estudantes de PósGraduação em Biblioteconomia também destacaram as funções "aprendizagem" e "lazer" em relação às demais. Na seqüência, os autores relatam como funções mais identificadas: "utilidade", "alvos sociais definidos", "estímulo" e "flexibilidade".

É interessante assinalar que foram priorizadas, tanto em Lopes e col. (1992) como em Oliveira (1993), as funções "aprendizagem", "lazer" e "utilidade", porém, a ocorrência das duas últimas foi inversa nos dois estudos, o que sugere que é preciso considerar as características dos cursos pesquisados.

Pode-se, por conseguinte, supor que as funções de leitura identificadas pelos estudantes universitários definem suas necessidades intelectuais, estéticas e de auto-atualização e, conseqüentemente, sua maturidade como leitor.

Considerando-se que a definição do objetivo da leitura organiza o raciocínio para a compreensão do texto, a consciência do motivo da leitura propicia o desenvolvimento do interesse que favorece um espírito aberto às modificações, à crítica e à criatividade, pontos fundamentais na formação de um leitor habilitado.

Dada a importância da leitura na formação pessoal e profissional do estudante universitário, este estudo teve como objetivos:

- descrever as funções de leitura identificadas pelos estudantes de Graduação;

- descrever as funções de leitura identificadas pelos estudantes de Pós-Graduação e

- comparar os dois grupos com referência ao uso das diferentes funções.

\section{Método}

\section{Sujeitos}

Participaram desta pesquisa um total de 25 sujeitos, dos quais 11 eram alunos do curso de pós-graduação em Psicologia Escolar da PUCCAMP e 14 eram alunos de graduação das Faculdades Integradas São Camilo. Dos alunos da graduação, cinco estavam matriculados e freqüentavam o $1^{\circ}$ ano do curso de Fonoaudiologia e nove, $\mathrm{o} 1^{\circ}$ ano do curso de Matemática.

Do total de sujeitos, $72 \%$ eram do sexo feminino e $28 \%$ do sexo masculino. A maior porcentagem de elementos do sexo feminino também foi observada nos cursos de pósgraduação $(54,5 \%)$ e de graduação $(85,7 \%)$.

A faixa etária dos sujeitos focalizados neste estudo situou-se entre 17 e 54 anos. Entre os estudantes de pós-graduação, a amplitude de idade situou-se entre 22 e 54 anos, com uma média de 31 anos. Na graduação, a faixa etária variou entre 17 e 37 anos, com uma média de 21 anos.

\section{Material}

$\mathrm{O}$ instrumento de pesquisa utilizado para a coleta de dados foi a Escala de Funções da Leitura, proposta por Greaney e Newman (1990) e traduzida e ampliada por Witter (1990), tendo sido pré-testada anteriormente e usada em pesquisas (no prelo).

Inicialmente, foram solicitados os dados de identificação dos sujeitos, referidos por nome, idade, profissão, escolaridade e sexo.

A escala é constituída de 50 itens organizados em função das dez funções da leitura identificadas por Greaney e Newman (1990). Para cada item, o sujeito deveria responder de acordo com uma escala de avaliação correspondente a muito, mais ou menos, pouco ou nada. 


\section{Procedimento}

A coleta de dados foi realizada pelos pesquisadores que utilizaram a situação de sala de aula. A escolha dos sujeitos foi assistemática. O mesmo procedimento foi utilizado para pósgraduandos e graduandos: os sujeitos responderam individual e imediatamente, por escrito, na própria folha apresentada pelos pesquisadores. Não foi delimitado tempo para a realização da atividade.

\section{Resultados e discussão}

As funções da leitura identificadas pelos sujeitos da graduação (GG) e pelos da pósgraduação (GP) estão resumidas na Tabela 1.

Os itens que apresentaram porcentagem acima de $50 \%$ na referência muito, para os dois grupos, são os 3, 9 e 24 relativos à função Aprendizagem; os itens 5, 10 e 13 são relativos à função Lazer ; já para a função Alvos Sociais Definidos são os 14 e 18 e para Utilidade são os 7 e 47.

Entre 50 e $20 \%$ na opção muito encontramos, ainda para os dois grupos, o item 1 para Lazer, 4 e 42 para Flexibilidade, 8 para Fuga, 12, 19 e 30 para Estímulo, 20 para Aprendizagem e 22 e 35 para Utilidade.

Os itens com referência muito abaixo de $10 \%$ foram os de número 11,39 e 48 para a função Preencher o Tempo; 15, 32 e 34 para Auto-Respeito; 16 e 23 para Fuga; 17 para Aprendizagem; 27 para Flexibilidade; 39 para Preencher o Tempo; 33 para Moralidade e 46 para Alvos Sociais Definidos.

Tabela 1. Percentual das Funções da Leitura identificadas pelos dois grupos GP- GG.

\begin{tabular}{|c|c|c|c|c|c|c|c|c|}
\hline \multirow[t]{2}{*}{ Afirmativa } & \multicolumn{2}{|c|}{ Muito } & \multicolumn{2}{|c|}{ Mais ou menos } & \multicolumn{2}{|c|}{ Pouco } & \multicolumn{2}{|c|}{ Nada } \\
\hline & GP & GG & GP & GG & GP & GG & GP & GG \\
\hline 1- Leio porque é excitante & 27,2 & 21,4 & 72,7 & 57,1 & 0 & 21,4 & 0 & 0 \\
\hline $\begin{array}{l}\text { 2-Leio porque me faz sentir como se estivesse indo para outro mundo e } \\
\text { tendo uma aventura. }\end{array}$ & 45,4 & 28,5 & 45,4 & 35,7 & 9,0 & 14,2 & 0 & 21,4 \\
\hline 3- Leio porque me ajuda na escola ou no trabalho & 100 & 64,2 & 0 & 35,7 & 0 & 0 & 0 & 0 \\
\hline 4- Leio porque acho fácil & 36,3 & 21,4 & 45,4 & 50,0 & 18 & 14,2 & 0 & 14,2 \\
\hline 5- Leio porque é interessante & 80,0 & 84,6 & 20,0 & 7,6 & 0 & 7,6 & 0 & 0 \\
\hline 6- Leio para saber mais sobre minha religião & 72,7 & 7,1 & 27,2 & 14,2 & 0 & 50 & 00 & 28,5 \\
\hline 7- Leio porque me ajuda com os assuntos que irei estudar mais tarde & 54,5 & 57,1 & 45,4 & 21,4 & 0 & 21,4 & 0 & 0 \\
\hline 8- Leio para tirar coisas (preocupações) de minha cabeça & 18,1 & 35,7 & 36,3 & 21,4 & 18,1 & 14,2 & 27,2 & 28,5 \\
\hline 9- Leio porque me faz compreender mais & 80,0 & 84,6 & 20,0 & 7,6 & 0 & 7,6 & 0 & 0 \\
\hline 10- Leio porque sinto prazer na leitura & 72,7 & 50,0 & 18,1 & 35,7 & 9,0 & 14,2 & 0 & 0 \\
\hline 11- Leio para acabar com a solidão & 0 & 0 & 27,2 & 28,5 & 27,2 & 7,1 & 45,4 & 64,2 \\
\hline 12- Leio para por pensamentos em minha cabeça & 18,1 & 15 & 318,1 & 38,4 & 54,5 & 15,3 & 9,0 & 30,7 \\
\hline 13- Leio porque os livros trazem boas histórias & 54,5 & 50,0 & 27,2 & 35,7 & 9,0 & 14,2 & 9,0 & 0 \\
\hline 14- Leio porque me ajudará a ter um bom trabalho & 63,6 & 57,1 & 27,2 & 28,5 & 0 & 14,2 & 9,0 & 0 \\
\hline 15-Leio porque meus pais (ou superiores) pensam que é importante & 0 & 7,1 & 36,3 & 0 & 18,1 & 14,2 & 45,4 & 78,5 \\
\hline
\end{tabular}




\begin{tabular}{|c|c|c|c|c|c|c|c|c|}
\hline \multirow[t]{2}{*}{ Afirmativa } & \multicolumn{2}{|c|}{ Muito } & \multicolumn{2}{|c|}{ Mais ou menos } & \multicolumn{2}{|c|}{ Pouco } & \multicolumn{2}{|c|}{ Nada } \\
\hline & GP & GG & GP & GG & GP & GG & GP & GG \\
\hline 16- Leio porque me ajuda a esquecer meus aborrecimentos & 0 & 7,1 & 18,1 & 28,5 & 36,3 & 28,5 & 45,4 & 35,7 \\
\hline 17- Leio para saber como as pessoas vivem em outros países & 18,1 & 0 & 45,4 & 35,7 & 27,2 & 42,8 & 9,0 & 21,4 \\
\hline 18- Leio para progredir nos meus estudos (ou carreira) & 90,9 & 57,1 & 9,0 & 42,8 & 0 & 0 & 0 & 0 \\
\hline 19-Leio porque é divertido pensar que sou um personagem da história & 27,2 & 28,5 & 9,0 & 21,4 & 45,4 & 21,4 & 18,1 & 28,5 \\
\hline 20- Leio para aprender a respeito de outras pessoas & 36,3 & 35,7 & 36,3 & 21,4 & 18,1 & 35,7 & 9,09 & 7,1 \\
\hline 21- Leio porque me ajuda a dormir facilmente & 0 & 21,4 & 0 & 28,5 & 45,4 & 21,4 & 54,5 & 28,5 \\
\hline 22-Leio para passar nos exames & 27,2 & 28,5 & 36,3 & 50,0 & 27,2 & 7,1 & 9,0 & 14,2 \\
\hline 23- Leio quando sinto solidão & 9,0 & 7 & 19,0 & 14,2 & 36,3 & 21,4 & 45,4 & 57,1 \\
\hline 24- Leio porque ajuda aprender algo novo & 90,9 & 76,9 & 9,0 & 23,0 & 0 & 0 & 0 & 0 \\
\hline 25-Leio porque me faz feliz & 54,5 & 23,0 & 18 & 138,4 & 27,2 & 30,7 & 0 & 7,6 \\
\hline 26- Leio porque me faz pensar um mundo melhor & 63,3 & 23,0 & 27,2 & 38,4 & 0 & 15,3 & 9,0 & 23,0 \\
\hline 27- Leio quando năo há nada de excitante na TV & 0 & 7,6 & 9,0 & 30,7 & 54,5 & 15,3 & 36,3 & 46,1 \\
\hline 28- Leio quando não sou forçado a fazê-lo & 0 & 61,5 & 36,3 & 7,6 & 18,1 & 7,6 & 45,4 & 23,0 \\
\hline 29- Leio porque o livro é um amigo quando estou só & 18 & 123,0 & 36,3 & 15,3 & 18,1 & 23,0 & 27,2 & 38,4 \\
\hline 30- Leio porque assim posso imaginar outra época da história & 27,2 & 30,7 & 96,3 & 30,7 & 27,2 & 15,3 & 9,0 & 23,0 \\
\hline 31- Leio porque me ajuda a saber o que é certo e o que é errado & 18,1 & 30,7 & 36 & 330,7 & 18,1 & 23,0 & 27,2 & 15,3 \\
\hline 32-Leio porque as pessoas me recompensam por ser bom leitor & 10,0 & 0 & 10,0 & 23,0 & 40,0 & 15,3 & 40,0 & 61,5 \\
\hline 33- Leio porque os livros me mostram o meio correto de viver & 0 & 0 & 63,6 & 23,0 & 9,0 & 46,1 & 27,2 & 30,7 \\
\hline 34- Leio para me tornar uma pessoa importante & 9,0 & 0 & 36,3 & 38,4 & 18,1 & 15,3 & 36,3 & 46,1 \\
\hline 35- Leio porque me ajuda a aprender mais palavras & 45,4 & 46,1 & 36,3 & 38,4 & 9,0 & 7,6 & 9,0 & 15,3 \\
\hline 36- Leio porque me ajuda a viver melhor & 27,2 & 15,3 & 45,4 & 38,4 & 18,1 & 30,7 & 9,0 & 15,3 \\
\hline 37- Leio por ser agradável quando estou só & 18,1 & 23,0 & 27,2 & 15,3 & 27,2 & 30,7 & 27,2 & 30,7 \\
\hline 38- Leio porque será bom para meu pais se eu souber mais & 40,0 & 7,6 & 10 & 053 & 830,0 & 15,3 & 20,0 & 23,0 \\
\hline 39- Leio para matar o tempo & 0 & 8,3 & 9,0 & 33,3 & 36,3 & 16,6 & 54,5 & 41,6 \\
\hline 40- Leio porque é agradável pensar sobre as coisas que li & 54,5 & 46,1 & 36 , & 330,7 & 9,0 & 23,0 & 0 & 0 \\
\hline 41- Leio porque me ajuda a melhorar a escrita & 90,9 & 46 & 19,0 & 23,0 & 0 & 23,0 & 0 & 7,6 \\
\hline $\begin{array}{l}\text { 42- Leio porque é uma coisa que posso fazer tão rápido ou tão lenta- } \\
\text { mente quanto eu goste }\end{array}$ & 36,3 & 38,4 & 54,5 & 38,4 & 9,0 & 15,3 & 0 & 7,6 \\
\hline 43- Leio porque me ajuda a relaxar & 18,1 & 38,4 & 36,3 & 38,4 & 36,3 & 7,6 & 9,0 & 15,3 \\
\hline 44- Leio porque faz o tempo passar & 0 & 25,0 & 20,0 & 8,3 & 40,0 & 50,0 & 30,0 & 16,6 \\
\hline $\begin{array}{l}\text { 45- Leio porque meu professor (supervisor/orientador) acha que é im- } \\
\text { portante }\end{array}$ & 18,1 & 15,3 & 45,4 & 7,6 & 27,2 & 15,3 & 9,0 & 61,5 \\
\hline $\begin{array}{l}\text { 46- Leio porque o governo de meu país deseja que eu seja um leitor } \\
\text { competente }\end{array}$ & 9,0 & 7,6 & 0 & 7,6 & 18,1 & 15,3 & 72,7 & 69,2 \\
\hline 47- Leio para fazer melhor meu trabalho & 81,8 & 53,8 & 9,0 & 46,1 & 0 & 0 & 9,0 & 0 \\
\hline 48- Leio porque não tenho mais nada para fazer & 0 & 0 & 9,0 & 25,0 & 27,2 & 8,3 & 63,6 & 66,6 \\
\hline 49- Leio para saber ajudar meu país & 27,2 & 0 & 36,3 & 30,7 & 27,2 & 30,7 & 9,0 & 38,4 \\
\hline 50. Leio para merecer o respeito de todos & 27,2 & 15,3 & 27,2 & 30,7 & 27,2 & 23,0 & 18,1 & 30,7 \\
\hline
\end{tabular}


Entre 50 e $80 \%$, na mesma referência para os dois grupos, tem-se 7 para Utilidade, $10 \mathrm{e}$ 13 para Lazer e 14 para Alvos Sociais Definidos.

Para os Pós-Graduandos, acima de $80 \%$, foram assinalados os itens 3 e 24 para a função Aprendizagem, 18 para Alvos Sociais Definidos e 41 e 47 para Utilidade. Já para os graduandos, não foi identificada nenhuma função. Acima de $50 \%$, para os Pós-Graduandos, tem-se os itens 6 e 26 para Moralidade, 25 para Lazer, 40 para Estímulo, 41 para Utilidade. Os Graduandos apresentaram apenas o item 28 relativo à função Flexibilidade.

Abaixo de $10 \%$ tem-se, para os PósGraduandos, os itens 21 para Flexibilidade e 44 para Preencher o Tempo e, para os Graduandos, 38 e 49 para Alvos Sociais Definidos, 17 para Aprendizagem e 6 para Moralidade.

Entre 50 e 20\%, para os Pós-Graduandos estão os itens 36 (Moralidade), 49 (Alvos Sociais Definidos) e 50 (Auto-Respeito). Para os Graduandos, ficam 8 e 29 (Fuga), 21 (Flexibilidade), 25 (Lazer), 26 e 31 (Moralidade), 37 (Fuga) e 43 e 44 (Preencher o Tempo).

Na Fig.1 estão representadas as médias das funções de leitura segundo os dados obtidos nos dois grupos (GG e GP).

As funções predominantes nos dois grupos de sujeitos foram Aprendizagem $(\mathrm{GP}=12,18$ e $\mathrm{GG}=10,92) ;$ Utilidade $(\mathrm{GP}=12,0 \mathrm{e}$ $\mathrm{GG}=10,64)$ e Lazer $(\mathrm{GP}=11,90$ e $\mathrm{GG}=10,78)$. Ainda considerando os dois grupos, a função que apresentou menor índice médio foi Preencher o Tempo $(\mathrm{GP}=4,45$ e GG=4,92).

Observa-se que as funções Estímulo e $\mathrm{Al}$ vos Sociais Definidos aparecem com bom índice de identificação média para os dois grupos $(\mathrm{GP}=9,54$ e $\mathrm{GG}=7,85 ; \mathrm{GP}=9,36$ e $\mathrm{GG}=7,71$, respectivamente).

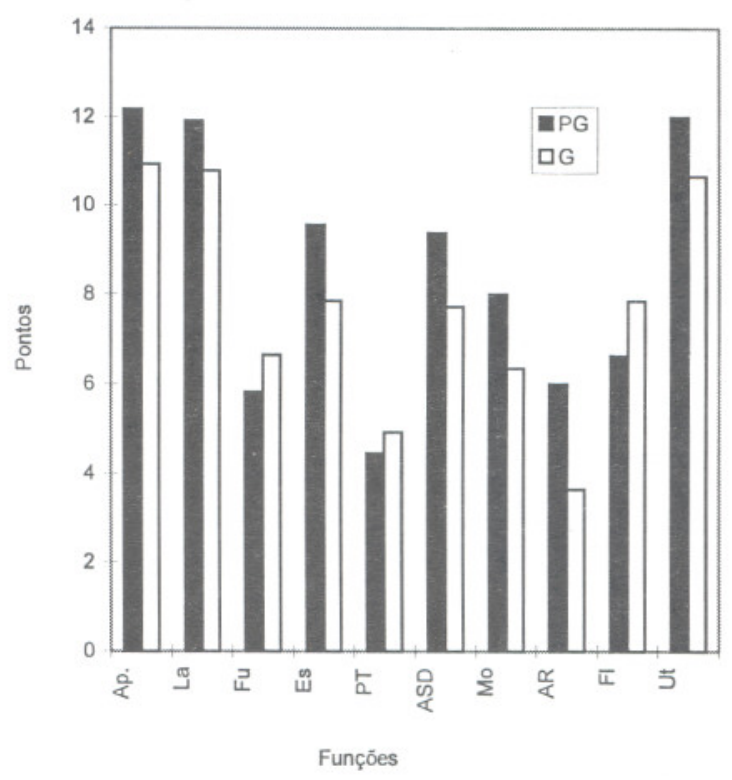

Fig.01 Funçōes da Leitura nos Grupos de Graduação e Pós graduação

Com valores situados entre 8 e 5 estão relacionadas as funções Fuga, Moralidade e Flexibilidade.

A função Auto-Respeito apresentou um resultado diferente em relação à média de identificação, considerando-se a diferença existente entre os dois grupos. Enquanto para o grupo de Pós-Graduandos encontrou-se um índice igual a 6, para o grupo de graduandos o índice encontrado situa-se na faixa de 3 pontos.

Para a verificação do grau de significância da distribuição de uso das funções para os dois grupos foi utilizada a prova U de Mann e Whitney (1956). Como hipótese básica $\left(\mathrm{H}_{\mathrm{O}}\right)$, considera-se que a utilização das funções independe do curso ao qual o aluno está vinculado; como hipótese alternativa $\left(\mathrm{H}_{1}\right)$, que a utilização das funções depende do curso freqüentado pelo sujeito. O nível de significância foi de 0,05 ( Tabela 2). O resultado indica que não há diferença significante entre os dois grupos. 
Tabela 2. Grau de significância da distribuição das funções para GM e GF. objetividade inerente ao curso. Inversamente, a área de humanas exercita um relacionamento menos pragmático e mais reflexivo, o que pode levar a maior identificação da função Lazer como um motivo de leitura (Lopes, 1992).

É de se esperar de bons leitores a utilização de outras funções como Estímulo, Moralidade e Alvos Sociais Definidos. Segundo Greaney (1990), as funções variam de acordo com as regiões nas quais o sujeito vive. Aquelas mais desenvolvidas, formam leitores conscientes de seu papel cívico e, portanto, retratam uma leitura voltada ao conhecimento do povo, do governo e dos fatos que ocorrem, permitindo a formulação

Do ponto de vista social e pessoal, a análise das funções atribuídas à leitura por um extrato da população permite identificar a disponibilidade destes ao intercâmbio de idéias, planejamento, desenvolvimento e formulação de princípios, enfim, permite avaliar o grau de maturidade do leitor (Oliveira, 1993).

Ao assumir a posição de sujeito-leitor atualizam-se alguns motivos psicológicos, entre eles, auto-respeito, auto-realização, segurança e sucesso. Com base nestes dados, pode-se explicar e justificar a utilização da leitura atendendo as funções Aprendizagem e Utilidade.

Estes resultados compatibilizam-se com estudos anteriores (Greaney, 1990; Lopes, 1992; Oliveira, 1993) e não só reforçam a hipótese acima, mas também justificam as diferenças próprias de cada curso e área de conhecimento. No presente estudo e no de Oliveira (1993), que tiveram entre seus sujeitos, estudantes da área de exatas, observou-se junto com a função Aprendizagem um alto índice para a função Utilidade, que pode estar relacionada à de julgamentos objetivos e críticos (Stauffer, 1969). Atendendo a este objetivo, as funções mencionadas voltam-se à formação do leitor como cidadão, a qual envolve o desenvolvimento de conceitos éticos e morais e também religiosos.

No presente estudo, o índice relativo à função Auto-Respeito marca um baixo nível de utilização por parte dos sujeitos, o que não seria esperado quando se considera que ela atende a uma necessidade básica para a formação do cidadão. Portanto, esta função deveria situar-se em nível de utilização compatível com a expectativa profissional proposta pelos diferentes cursos.

A Flexibilidade, definida como a função que garante ao sujeito a possibilidade de leituras diversificadas e, ao mesmo tempo, a identificação e a escolha de diferentes tipos de suportes, identifica a maturidade do leitor. Assim, era de se esperar dos sujeitos aqui pesquisados um alto uso desta função; o que não ocorreu. Este fato permite questionar sua habilidade como leitores ao mesmo tempo que sugere a 
realização de uma pesquisa visando identificar seu comportamento de leitura.

As funções Preencher o Tempo e Fuga podem ter sido rejeitadas por sua "tonalidade negativa” (Greaney, 1990). Porém, a análise das afirmações possíveis a estas funções identifica a possibilidade do afastar-se da vida moderna assumindo postura reflexiva e avaliativa sobre si mesmo e sobre o texto (Oliveira, 1993). Esta atitude permite ao leitor sua valorização como tal e, conseqüentemente, lhe garante a autonomia e a independência como aprendiz.

A Universidade, como o próprio nome indica, deve preocupar-se com uma formação humanística global, mais abrangente e mais conscientizadora. Ela não pode se fundamentar no repassar conhecimento. Deve, ao contrário, propor um processo de ensino e aprendizagem voltado para um desempenho crítico e independente, atendendo à formação do aluno como cidadão.

Entretanto, já é conhecido (Pugh \& Pawan, 1991) que a preparação dos alunos na Universidade está crítica. Este fato parece concretizar-se no presente trabalho em que se observou que não houve diferença significante no uso das funções pelos estudantes graduandos e pós-graduandos, o que pode suscitar a questão sobre a atuação desta instituição na formação do aluno.

Para que se possa reverter tal processo, deve-se buscar conhecer e descrever a população que atua na Universidade, suas competências e suas limitações. Deve-se, também, considerar a necessidade de se analisar os currículos dos diferentes cursos e, com base nessa análise, elaborar estratégias de habilitação e reabilitação de leitura a fim de se garantir a formação do aprendiz independente e autônomo.

\section{Conclusão}

O grau de maturidade de um leitor pode ser identificado pelo índice de utilização das funções da leitura, pois ao assumir este papel, espera-se que sejam atualizados alguns motivos psicológicos, como auto-respeito, autorealização, segurança e sucesso. Estes fatos podem justificar as diferenças encontradas nos estudantes dos cursos focalizados no presente estudo.

- As funções mais identificadas pelos sujeitos foram Aprendizagem, Utilidade e Lazer e a menos utilizada foi Preencher o Tempo, para os dois grupos.

- A função Auto-Respeito, com baixo nível de identificação para os dois grupos, retrata uma situação não-compatível com o status de formação dos sujeitos. Na medida em que ela atende a uma necessidade básica para a formação do cidadão, era de se esperar que o grupo de estudantes de pós-graduação estivesse mais voltado para este aspecto formativo se comparado àquele de graduação.

- Na mesma linha de análise, seria esperado, também, que a função Flexibilidade aparecesse de forma mais evidente no grupo de pós-graduandos, pois ela identifica a maturidade na escolha dos suportes e dos tipos de texto de leitura.

- A análise da distribuição de uso das funções para os dois grupos não mostrou diferença significante entre eles.

Os dados obtidos neste estudo evidenciam a necessidade de novas pesquisas visando fortalecer o conhecimento sobre o leitor adulto e, conseqüentemente, orientar para a reformulação e a proposição de formas de orientação e estratégias curriculares de construção e aprimoramento do estudante/leitor. 


\section{Referências}

Carnio, M.S.(1995). Conceitos e Compreensão de Leitura e Escrita do Surdo no Contexto da Educação Especial. Tese de Doutoramento. São Paulo:USP.

Dolly, M.R. (1990). Integrating ESL reading and writing through authentic discourse. Journal of Reading, 33 (5): 360-365.

Fidalgo, F.S. (1994). Qualidade e Novas Tecnologias em Educação. Encontro Nacional dos Estudantes do Ensino Superior das Escolas Particulares. São Paulo: CONTEE.

Field, M.L.; Aebersold, J.A. (1990). Cultural attitudes toward reading implications for teachers of ESL/Bilingual readers. Journal of Reading, 33 (6): 406-410.

Granja, E.C. (1985). Contribuições ao estudo da leitura entre estudantes universitários. Dissertação de Mestrado. São Paulo: USP.

Gray, W.S. (1984). Reading. Newark, Del: IRA.

Greany, V.; Newman, S.B. (1990). The function of reading a cross cultural perspective. Reading Research Quartely, 26 (3): 172-195.

Lopes, T. M. J.; Ribeiro, M. S. P. (1992). Funções da leitura entre pós-graduandos em biblioteconomia. Trans-in-formação, 4 (1,2,3): 45-54.

Oakhill, J. and Garnham, A. (1988). Becoming a skilled reader. New York: Basil Blackeell.

Oliveira, M. H. M. A. (1993). A leitura do universitário: estudo comparativo entre os cursos de engenharia e fonoaudiologia da PUCCAMP. Dissertação de Mestrado. Campinas: PUCCAMP.

Pugh, S.; Pawan, F. Reading and Writing and Academic Litercy. Flippo, R. \& Carvely, D. (1991). College Reading \& Study Strategy Programs. Newark Delaware, International Reading Association.

Santos, A.A.A. (1990). Leitura e Universidade: uma análise de algumas questões críticas. Trans-informação, 2 (2/3): 91-104.
Stauffer, R.G. (1969). Directing Reading Maturity as a Cognitive Process. Harper and Row, Publishers, N.Y.

Wepner, S.H.; Felley, J.T.; Minery, B. (1990). Do computers have a place in college reading courses? Journal of Reading, 33 (5):348-349.

Witter, G. P. (1990). Pesquisa bibliográfica, pesquisa documental e busca de informação. Estudos de Psicologia, 7 (1): 5-30. 